\title{
Locally Advanced Gareinoma Esophagus with Right Nipple Metastasis
}

\section{JCR}

\author{
Ajaz Ahmad Malik, Manish K. Singhal \\ Department of Medical Oncology Fortis International Oncology, Noida NCR, India.
}

\section{Abstract:}

Squamous cell carcinoma of the esophagus is the most common histological subtype of cancer in the upper and middle esophagus and it accounts the sixth most common cause of cancer related deaths worldwide. The incidence of esophageal cancer occurs at a high frequency in Asia and South America. We report 52 year old male having carcinoma esophagus with nipple metastatsis.

Key words: Adenocarcinoma, Esophageal Neoplasms, Melanoma, Nipples, Squamous Cell Carcinomas.

\section{Introduction}

Patients with esophageal cancer usually present with locally advanced disease which have metastasized to other organs at the time of diagnosis. There are two main forms of esophageal cancer with different etiological and pathological characteristics: esophageal squamous cell carcinoma (ESCC) and esophageal adenocarcinoma (EADC); predominant histological subtype being ESCC. Skin metastasis from esophageal squamous cell carcinoma is rare with frequency of $0.7 \%$ and $9 \%$ [1]. In clinical practice, skin metastasis from the underlying cancer are uncommon, but once detected, indicate the diagnosis of some internalmalignancy. There is limited review in the recent literature. Skin metastasis from carcinoma breast, lung and melanomas are common but esophageal cancers seldom metastasize to skin $[2,3]$. Metastatic spread to skin occurs via lymphatic or hematogenous route, presents in form of papules or nodules, and survival rate is 4 to 5 months [4-6].
We report a case of 52 year old male having carcinoma esophagus with right nipple metastasis.

\section{Case Report}

A 52 years male patient presented with complaints of severe dysphagia of 3 months duration. UGI endoscopy done outside was suggestive of ulcerated growth in esophagus at $25 \mathrm{~cm}$ from incisors. Biopsy was suggestive of squamous cell carcinoma. CECT neck and thorax was suggestive of short segmental wall thickening of mid esophagus on right lateral aspect with focal partial irregular luminal narrowing and proximal dilatation. Necrotic nodes approximately $2.5 \mathrm{~cm}$ were seen in right paratracheal and subcarinal regions. Subsequently, patient was referred to us for further evaluation. Cutaneous examination showed a skin lesion $1 \mathrm{~cm}$ at right nipple and was suspected to be metastatic or infective [Fig.1].

\section{Corresponding Author: Dr. Ajaz Ahmad Malik}

Email: ejreen@gmail.com

Received: October 26, 2015 | Accepted: March 21, 2016 | Published Online: May 10, 2016

This is an Open Access article distributed under the terms of the Creative Commons Attribution License (creativecommons.org/licenses/by/3.0)

Conflict of interest: None declared | Source of funding: Nil | DOI: http://dx.doi.org/10.17659/01.2016.0050 
PET CT scan was suggestive of heterogeneously enhancing circumferential neural thickening with mass formation in midthoracic esophagus causing luminal compromise with metastatic right supraclavicular, mediastinal and paraesophageal lymphadenopathy; metastatic subcentimeter subcutaneous enhancing soft tissue lesion in right chest wall. FNAC from nipple lesion confirmed metastatic squamous cell carcinoma [Fig.2,3]. After complete evaluation he was started on chemotherapy.

Early recognition of tumor relapse from a suspicious skin lesion may lead to initiation of treatment before widespread metastases occur. In our case, the nipple metastasis was found simultaneously with the primary esophagus tumor, facilitating diagnosis. However the patient died after 1 year of receiving treatment

\section{Discussion}

Generally, cutaneous metastases are early indicators of metastatic disease. Diagnosis may be delayed by several months, unless the skin lesion grows rapidly or other sites such as the lung or liver are affected by the tumor spread.

There is only limited data available in this case due to rarity of skin metastasis from squamous carcinoma esophagus. The most common sites of hematogenous metastases from esophageal carcinoma are the lungs and liver whereas the less frequently affected sites are the bones [7]. A study conducted by Lookingbill et al. [8] in 7,316 patients with skin cancer and skin metastasis, none of the patient was detected to have primary carcinoma esophagus. The most common cancer having skin metastases are carcinoma breast and melanomas. Skin metastasis is diagnosed based on histopathology and often is faced with lots of challenges. The detection of skin metastasis in

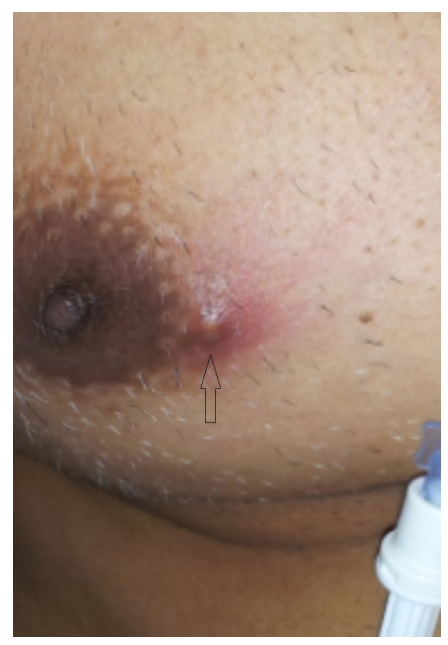

Fig.1: Metastatic skin lesion at right nipple.

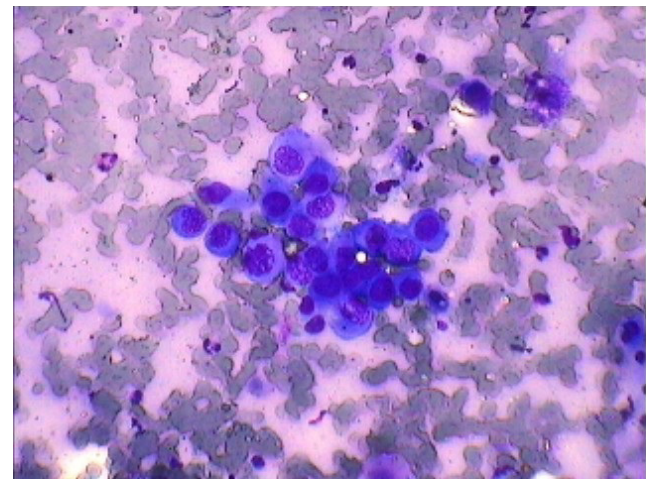

Fig.2: HPE nipple lesion with clusters of squamous cells.

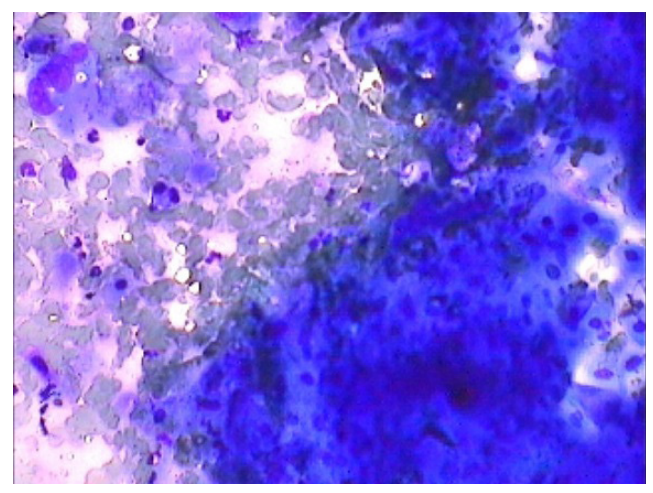

Fig.3: $2^{\text {nd }}$ HPE of the nipple, with clusters of squamous cell. 
carcinoma esophagus is a signal of poor prognosis. The presence of skin metastasis in ESCC (esophageal squamous cell cancer) is very rare and is rarely reported. Skin metastasis are most of the time painless and can be confused with papules.

Studies have revealed the overall survival time is approximately 4 to 5 months after being detected to have skin metastasis [4]. In our case the nipple lesion initially looked like a papule and was thought to be the infective lesion, however FNAC proved it to be metastatic. He initially responded well to the chemotherapy but chemotherapy had no effect on his nipple metastasis and it remained of the same size during his entire course of chemotherapy. He even did not respond to $2^{\text {nd }}$ line chemotherapy and after counseling the family was kept on palliative care. Therefore, in the case of sharply demarcated and painless papule of the skin which progresses rapidly a cutaneous metastasis of an unknown primary tumor should be considered in the differential diagnosis. Biopsy for early histological examination should be performed on any suspicious skin lesion. Subsequent positron emission tomography (PET) should be considered in all patients as part of the initial work up for the primary tumor as done in our case.

As newer combined-modality treatments, such as neoadjuvant chemoradiation followed by surgical resection, and newer cytotoxic agents became available, loco-regional disease recurrence has been dramatically reduced. Today, in most patients who have undergone combinedmodality treatments for advanced esophageal carcinoma, recurrences have mainly been from hematogeneous metastatic spread. Patients may present with distant metastasis much earlier than they develop local recurrence in form of dysphagia/weight loss. A complete history, careful physical examination, and radiology studies (CT scans, bone scans, positron-emission tomography) are essential in assessment of distant failures which suggests poor prognosis [9].

\section{Conclusion}

Skin manifestations of ESCC are extremely rare and only a small number of cases with solid skin metastases have been reported. A case of ESCC with such diffuse and massive skin metastases, most likely indicating highly aggressive disease, has not been described previously.

\section{Acknowledgements}

We thank Dr Rajeev Tangri, Consultant HPE for providing us the histopathology pictures

\section{References}

1. Levine MS, Halvorsen RA. Carcinoma of the esophagus. In: Gore RM, Levine MS, editor. Textbook of Gastrointestinal Radiology. Philadelphia, PA: Saunders; 2000. pp. 403433.

2. Brownstein $M H$, Helwig EB. Patterns of cutaneous metastasis. Arch Dermatol. 1972;105:862-868.

3. Schoenlaub P, Meyer P, Heid E, Grosshans E, Cribier B. Métastases cutanées révélatrices d'un cancer méconnu. Étude anatomoclinique de 40 cas. Ann Dermatol Venereol. 1998;3S:90-91.

4. Schoenlaub P, Sarraux A, Grosshans E, Heid $E$, Cribier B. Survival after the occurrence of cutaneous metastasis: a study of 200 cases. Ann Dermatol Venereol. 2001;128:1310-1315.

5. Lookingbill DP, Spangler N, Helm KF. Cutaneous metastases in patients with metastatic carcinoma: A retrospective study of 4020 patients. J Am Acad Dermatol. 1993;29:228-236.

6. Schwartz RA. Cutaneous metastatic disease. J Am Acad Dermatol. 1995;31:161-182.

7. Quint LE, Hepburn LM, Francis IR, et al. Incidence and distribution of distant metastases from newly diagnosed esophageal carcinoma. Cancer. 1995;76:1120-1125. 
8. Lookingbill DP, Spangler N, Sexton FM. Skin involvement as the presenting sign of internal carcinoma. J Am Acad Dermatol. 1990;22:19-26.
9. Maheshwari G, Kale N, Halder P. Unusual skin manifestations from squamous cell carcinoma of oesophagus. OMJ. 2010;25:51-52. 